May 2021

"Strategic information transmission with sender's approval"

Françoise Forges, Jérôme Renault 


\title{
Strategic information transmission with sender's approval
}

\author{
Françoise Forges* and Jérôme Renault ${ }^{\dagger}$
}

January $2020^{\ddagger \S}$

\footnotetext{
*PSL, Université Paris-Dauphine, LEDa. E-mail: francoise.forges@gmail.com

†TSE, Université de Toulouse 1, Département de Mathématiques.

${ }^{\ddagger}$ This research started during the winter 2015-2016, while the first author was visiting Humboldt University, Berlin. We did not know of Shimizu $(2013,2017)$ at the time. These papers were pointed out to the first author by Daniel Krähmer on the occasion of a talk in Bonn in April 2018. Then we discovered Matthews (1989), among the references of Shimizu $(2013,2017)$.

${ }^{\S}$ We thank Anna Bogomolnaia, Ulrich Horst, Vincent Iehlé, Frédéric Koessler, Daniel Krähmer, Ehud Lehrer, Ronny Razin, Antoine Salomon, Roland Strausz and Bertrand Villeneuve for stimulating conversations on the topic of this research. We also thank the participants of the workshops "Competition and Incentives" (Humboldt University, Berlin, June 9-10, 2016), "Game theory: Economics and Mathematics (GEM)" (South University of Denmark at Odense, October 5-6, 2018), "Signaling in Markets, Auctions and Games: a Multidisciplinary Approach" (Paris 2, May 22-23, 2019), "Frontiers in Design" (University College London (UCL), June 14-15, 2019), "Information Design and Splitting Games" (Centre d'Economie de la Sorbonne, Paris, June 17-19, 2019), as well as the audiences of seminars in Besançon, Bonn, Lancaster, London (LSE), Paris-Dauphine and Rome (LUISS). Jérôme Renault gratefully acknowledges funding from ANR-3IA Artificial and Natural Intelligence Toulouse Institute, grant ANR-17-EUR-0010 (Investissements d'Avenir program) and ANR MaSDOL.
} 


\begin{abstract}
We consider a sender-receiver game with an outside option for the sender. After the cheap talk phase, the receiver makes a proposal to the sender, which the latter can reject. We study situations in which the sender's approval is crucial to the receiver.

We show that a partitional, (perfect Bayesian Nash) equilibrium exists if the sender has only two types or if the receiver's preferences over decisions do not depend on the type of the sender as long as the latter participates. The result does not extend: we construct a counter-example (with three types for the sender and type-dependent affine utility functions) in which there is no mixed equilibrium. In the three type case, we provide a full characterization of (possibly mediated) equilibria.
\end{abstract}




\section{Introduction}

We consider a general model of sender-receiver games. The specific feature of our games is that the sender has an outside option. After the cheap talk phase, the receiver proposes a decision to the sender; if the sender approves it, the decision is made; otherwise, the sender chooses his outside option, which can be interpreted as "exit". Under complete information, the game reduces to an ultimatum game, in which one player makes a "take it or leave it" offer to the other. In our framework, this other player has private information and can send a costless message to the receiver before getting an offer.

We are interested in situations in which the sender's approval is crucial to the receiver. We thus assume that the receiver's utility in case of exit is very low, as compared to what he can expect if the sender accepts his proposal. It is not difficult to find examples in which a decision-maker consults with an informed party before making a proposal that can be ultimately rejected and in which rejection has unvaluable, damaging consequences for the decisionmaker. For instance, firms try to figure out workers' requirements in order to avoid strikes and boycotts. Governments discuss with kidnappers, hoping that hostages will not be killed. As a third example, analyzed in Matthews (1989), the U.S. Congress may worry about the President's veto.

We focus on equilibria in which the sender does not make uncredible threats at the approval stage, namely, accepts a proposal if and only if it gives him at least the utility of his outside option. ${ }^{1}$ We ask whether our sender-receiver game has an equilibrium in which exit does not occur.

To answer this question, we introduce an auxiliary "limit game" $\Gamma$, in which equilibria are necessarily without exit. The equilibria of $\Gamma$ are easily characterized by two sets of conditions: incentive compatibility and constrained optimization. Both sets of conditions are tractable but satisfying them jointly is demanding. Existence of an equilibrium in the game $\Gamma$ is not obvious. For instance, as opposed to standard sender-receiver games, $\Gamma$ may not have any nonrevealing equilibrium. This means that, in absence of information transmission, the receiver cannot make any decision that would give at least his reservation utility to the sender, whatever his type. We identify various assumptions which guarantee that, in a situation like this, the sender can credibly reveal some information to the receiver, in such a way that exit

\footnotetext{
${ }^{1}$ Our solution concept is basically subgame perfect Nash equilibrium. Except for the approval stage, our model behaves as a standard cheap talk game, in which Perfect Bayesian equilibrium is not restrictive.
} 
will never happen.

We maintain the following assumptions on the game $\Gamma$ : the sender has finitely many types (which can be multidimensional, e.g., belong to $\mathbb{R}^{n_{1}}$, for some $n_{1}$ ), the receiver has a compact set of decisions (typically, a closed, bounded set in $\mathbb{R}^{n_{2}}$, for some $n_{2}$ ) and both players' utility functions are continuous. $^{2}$ We also make the sine qua non assumption that under complete information, i.e., when the receiver knows the sender's type, there exists a decision that gives the sender at least his reservation utility. We then consider the subsets of all types such that there is a decision inducing them to participate and we call "participation structure" the maximal subsets (with respect to set inclusion). For instance, if the sender has only two possible types, 1 and 2, the participation structure is either $\{\{1\},\{2\}\}$ or $\{1,2\}$.

We establish that the game $\Gamma$ has a partitional equilibrium, namely, an equilibrium in which the sender's strategy is pure, in the following cases:

(i) the sender has two types;

(ii) the participation structure is a partition of the type set;

(iii) the decision set is a real interval and for every type, the sender's utility function is monotonic in the receiver's decision;

(iv) the receiver's utility function - when the sender participates - does not depend on the sender's type.

Cases (i) and (ii) are rather straightforward, with (ii) generalizing (i). Case (iii) applies in particular when the receiver has only two actions, over which he can randomize. Case (iv) has the most important scope. It applies as soon as the receiver knows his own preferences over decisions, but is eager to make a choice that will ensure the - type-dependent - informed player's participation. Existence of a partitional equilibrium under (iv) is the main result of the paper (Theorem 8).

The previous assumptions may look restrictive, but, without them, existence of an equilibrium in $\Gamma$ cannot be guaranteed, even if the sender is allowed to use a mixed strategy. We indeed propose an example, in which the sender has three types, the receiver has three actions, the participation structure is not a partition and the utility functions are type-dependent. In

\footnotetext{
${ }^{2}$ This covers the particular case where the receiver has finitely many actions, over which he can randomize.
} 
this example, there is no mixed equilibrium. However, an equilibrium does exist if the information transmission stage is handled by a mediator.

Finally, we propose a complete analysis when the sender has three possible types. We identify two kinds of "participation structures" beyond the straightforward case (ii) above. The first one arises in the example mentioned in the previous paragraph. Existence of a mediated equilibrium can then be established. In the other case, another example shows that there may not be any partitional equilibrium. However we prove that there always exists a mixed equilibrium if the decision set is convex and the utility functions are affine (Proposition 9).

Here is a description of the paper. We discuss the related literature below. In Section 2, we make the sender-receiver game $\Gamma$ and the solution concept fully precise. Propositions 3 and 4 (which are established in Section 6.1) allow us to argue that the game $\Gamma$ is relevant to our study. In Section 3, we establish existence of a partitional equilibrium in $\Gamma$ under assumptions (i), (ii), (iii) or (iv) above. Our main result, Theorem 8, is associated with case (iv). Section 4 is devoted to examples. Sections 4.1 and 4.2 propose a family of kidnapping games. Section 4.1 illustrates partitional equilibrium. Section 4.2 proposes a game that does not have any partitional equilibrium but has a mixed equilibrium. Section 4.3 goes on with a game that does not have any mixed equilibrium but has a mediated equilibrium. The three type case, including Proposition 9, is the topic of Section 5. Section 6 is an appendix containing the proofs of Propositions 3, 4 (Section 6.1) and 9 (Section 6.2).

\section{Related papers}

Shimizu (2013, 2017) adds an approval stage to Crawford and Sobel (1982)'s sender-receiver game, in the popular case where the prior is uniform over the unit interval and the utility functions are quadratic. He assumes, as we do, that exit is damaging for the receiver but the setup is otherwise quite different from ours. He shows that, in his particular model, credible exit possibilities can make cheap talk informative even when the players' conflict of interest is relatively large.

Matthews (1989) studies a sender-receiver game motivated by a specific application, in which the sender is the U.S. President, the receiver is the Congress and the decision is about a practical matter, like the level of military

expenditures. The President can veto the Congress' proposal. Preferences are unimodal, as in Shimizu $(2013,2017)$, but the receiver's utility does not depend on the sender's type (as in the current paper, Theorem 8, Section 
3.4). More importantly, in Matthews (1989)'s model, the sender's rejection leads to status quo, rather than to exit, and does not necessarily yield a very low utility to the receiver. Matthews (1989)'s point is to show that thanks to incomplete information on the President's type, veto can happen at equilibrium, i.e., without relying on uncredible threats.

Our model can be viewed as a principal-agent problem in which the principal - alias the receiver - cannot commit to a mechanism at the ex ante stage. This is an extreme case of Bester and Strausz (2001)'s principal-agent problem with limited commitment. In this context, it makes sense to allow the agent - alias the sender - to veto the principal's decision. Under a mechanism design perspective, the principal looks for an equilibrium that gives him the best ex ante expected utility, which amounts to an equilibrium in which all types accept the principal's proposal if the principal's utility, when the agent chooses his outside option, is sufficiently low. This leads us to impose individual rationality conditions for the agent at the "posterior" stage, i.e., after the principal has made a proposal. The relevance of posterior individual rationality and its impact on incentive compatibility have been stressed in a number of papers, e.g., Gresik (1991), Compte and Jehiel (2007, 2009) Forges (1990, 1999) and Matthews and Postlewaite (1989).

Finally, Forges and Horst (2018)'s concept "talk and cooperate (perfect Bayesian) equilibrium" (TCE, Section 5.3) is motivated by the same questions as the present paper, but is defined in a different model: the sender also has to make a decision, which is relevant to his own payoff only. At a TCE, the receiver (who can be interpreted as a principal) proposes a joint decision, which the sender accepts whatever his type. Should player 1 reject player 2's proposal, both players would choose an action, independently of each other. By contrast, in the present paper, the sender just chooses an outside option. Forges and Horst (2018) establish an existence result for another solution concept - "cooperate and talk (perfect Bayesian) equilibrium" (CTE) - but just indicate that their methodology does not apply to TCE.

\section{Model}

\subsection{Sender-receiver games}

We start with a family of games $\Gamma\left(v_{0}\right), v_{0} \in \mathbb{R}$, between a sender (player 1 ) and a receiver (player 2$). \Gamma\left(v_{0}\right)$ is described as follows: 
- A type $k \in K$ is chosen according to a prior probability $p \in \Delta(K)$.

- Player 1 is informed of $k$.

- Player 1 sends a message $m \in M$ to player 2 .

- Player 2 proposes a decision $x \in X$ to player 1 .

- If player 1 accepts player 2's proposal, the decision $x$ is enforced, player 1 gets $U^{k}(x)$ and player 2 gets $V^{k}(x)$.

- If player 1 rejects player 2's proposal, player 1 chooses an outside option and gets $u_{0}^{k}$. Player 2 gets $v_{0}$.

We assume that:

- The set of types $K$ is finite ${ }^{3}$ and $p^{k}>0 \forall k \in K$.

- The set of messages $M$ is finite, such that $|M| \geq|K|$.

- The set of decisions $X$ is compact. ${ }^{4}$ As a typical example, $X \subset \mathbb{R}^{n_{2}}$, for some $n_{2} \geq 1$; for instance, player 2 has a finite set of actions $A$ and $X=\Delta(A)$ corresponds to the set of mixed strategies of player 2 .

- The utility functions $U^{k}: X \rightarrow \mathbb{R}$ and $V^{k}: X \rightarrow \mathbb{R}$ are continuous; for instance, if $X=\Delta(A), U^{k}$ and $V^{k}$ may correspond to expected utility.

We further assume that:

- For every $k \in K$, there exists $x \in X$ such that $U^{k}(x) \geq u_{0}^{k}$.

- For every $k \in K$, for every $x \in X, V^{k}(x) \geq v_{0}$, namely,

$$
v_{0} \leq \min _{k \in K} \min _{x \in X} V^{k}(x) .
$$

\footnotetext{
${ }^{3}$ We do not make any assumption beyond the fact that there are finitely many types; in particular, types can be "multidimensional," with $K \subset \mathbb{R}^{n_{1}}$, for some $n_{1} \geq 1$.

${ }^{4}$ We will indicate explicitly when $X$ will be required to be convex.
} 
We are interested in situations in which the sender's approval is crucial to the receiver, namely, in which $v_{0}$ can be arbitrarily low. Let us denote as $\Gamma$ the "limit game," in which $v_{0}=-\infty$. We will show that $\Gamma$ is a tractable tool, which is appropriate to study $\Gamma\left(v_{0}\right)$ when $v_{0}$ is small enough.

Let us set, for every $L \subseteq K$

$$
X(L)=\left\{x \in X: U^{k}(x) \geq u_{0}^{k}, k \in L\right\} .
$$

Given a subset of types $L, X(L)$ is the set of decisions that are acceptable by all types in $L$. We write $X(k)$ for $X(\{k\})$, so that $X(L)=\bigcap_{k \in L} X(k)$.

\section{$2.2 \quad$ Equilibria}

Our solution concept, in $\Gamma\left(v_{0}\right)$ and $\Gamma$, is basically subgame perfect Nash equilibrium, but perfect Bayesian equilibrium would not be more demanding: as in standard sender-receiver games, finding beliefs rationalizing player 2's choices is not an issue. What is crucial here is to avoid non-credible threats from player 1 . In the sequel, we simply refer to "equilibrium."

At a subgame perfect Nash equilibrium, player 1 of type $k$ accepts (resp., rejects) player 2's proposal $x$ when $U^{k}(x)>u_{0}^{k}$ (resp., $U^{k}(x)<u_{0}^{k}$ ). We further assume that player 1 accepts the proposal when he is indifferent, which is consistent with our interest in situations in which player 2 strictly prefers that player 1 participates. By proceeding backwards, $\Gamma\left(v_{0}\right)$ amounts to a standard sender-receiver game, with the following utility functions (in which $I$ denotes the indicator function):

$$
U_{+}^{k}(x)=U^{k}(x) I\left(U^{k}(x) \geq u_{0}^{k}\right)+u_{0}^{k} I\left(U^{k}(x)<u_{0}^{k}\right)=\max \left\{U^{k}(x), u_{0}^{k}\right\}
$$

for player 1 of type $k$ and

$$
W^{k}\left(v_{0}, x\right)=V^{k}(x) I\left(U^{k}(x) \geq u_{0}^{k}\right)+v_{0} I\left(U^{k}(x)<u_{0}^{k}\right) .
$$

for player 2, when player 1 is of type $k .{ }^{5}$ In the latter sender-receiver game, the receiver's utility function is not necessarily continuous, but it is uppersemi-continuous.

\footnotetext{
${ }^{5}$ This observation is made in Chen, Nartik and Sobel (2008), in their account of Matthews (1989).
} 
Lemma 1. For every $k \in K$ and $v_{0} \in \mathbb{R}$, the utility function $W^{k}\left(v_{0}, \cdot\right)$ defined by (3) is upper-semi-continuous.

Proof: Let $x_{n} \in X, x_{n} \rightarrow x$. The only possibly delicate case is when $U^{k}\left(x_{n}\right)<u_{0}^{k}$ for every $n$ and $U^{k}(x)=u_{0}^{k}$. Then $W^{k}\left(v_{0}, x_{n}\right)=v_{0} \leq V^{k}(x)=$ $W^{k}\left(v_{0}, x\right)$, using our assumption.

Having determined player 1's behavior at the approval stage, we can define a strategy for player 1 (in $\Gamma\left(v_{0}\right)$ and $\Gamma$ ) as a mapping $\sigma: K \rightarrow \Delta(M)$. We interpret $\sigma(k)(m)$ as the probability that player 1 sends message $m$ when his type is $k$, and denote it as $\sigma(m \mid k)$. We adopt the following notations:

$$
\text { For every } m \in M, P_{\sigma}(m)=\sum_{k} p^{k} \sigma(m \mid k) \text {. }
$$

For every $k \in K$ and $m \in M$ s.t. $P_{\sigma}(m)>0, p_{m}^{k}(\sigma)=\frac{p^{k} \sigma(m \mid k)}{P_{\sigma}(m)}$.

$p_{m}^{k}(\sigma)$ is thus the posterior probability of type $k$ computed from $p$ and $\sigma$; let $p_{m}(\sigma)=\left(p_{m}^{k}(\sigma)\right)_{k \in K}$ denote the corresponding posterior probability distribution over $K$. We have $\sum_{m} P_{\sigma}(m) p_{m}(\sigma)=p$.

We say that $\sigma$ is nonrevealing if player 1 sends his message in a typeindependent way, namely, if $\sigma(m \mid k)=\sigma\left(m \mid k^{\prime}\right)$ for every $m \in M, k$, $k^{\prime} \in K$. In this case, $p_{m}(\sigma)=p$ for every $m$ s.t. $P_{\sigma}(m)>0$.

For player 2, a strategy is a mapping $\tau: M \rightarrow X$, namely, a "pure" strategy with respect to the set $X$ (but as indicated above, $X=\Delta(A)$ for a finite set of actions $A$ is a particular case). ${ }^{6}$

We say that $(\sigma, \tau)$ is "without exit" if

$$
U^{k}(\tau(m)) \geq u_{0}^{k} \forall k \in K, \forall m \in M \text { s.t. } \sigma(m \mid k)>0,
$$

namely, if

$$
U_{+}^{k}(\tau(m))=U^{k}(\tau(m)) \forall k \in K, \forall m \in M \text { s.t. } \sigma(m \mid k)>0 .
$$

Recalling (1) and denoting by supp $q$ the support of a probability distribution $q \in \Delta(K)$, condition (6) is equivalent to

$$
\tau(m) \in X\left(\operatorname{supp} p_{m}(\sigma)\right) \forall m \in M \text { s.t. } P_{\sigma}(m)>0 .
$$

\footnotetext{
${ }^{6}$ Restriction to pure strategies of player 2 is justified by the fact that these will be enough to establish existence of equilibria in $\Gamma$.
} 
At an equilibrium of $\Gamma$, we require that player 2's expected utility be finite $(>-\infty)$. Hence an equilibrium of $\Gamma$ is necessarily without exit. By contrast, an equilibrium of $\Gamma\left(v_{0}\right)$ may involve exit of some types.

Proposition 2. For every $v_{0} \in \mathbb{R}$, the game $\Gamma\left(v_{0}\right)$ has a nonrevealing equilibrium (possibly with exit). The game $\Gamma$ has a nonrevealing equilibrium if and only if $X(K) \neq \emptyset$. Hence $\Gamma$ may not have any nonrevealing equilibrium.

Proof: The following strategies define a nonrevealing equilibrium in $\Gamma\left(v_{0}\right)$ : player 1 sends the same message $m \in M$ whatever his type and then accepts $x$ if and only if $U^{k}(x) \geq u_{0}^{k}$; whatever the message, player 2 chooses $x^{*} \in X$ to maximize $\sum_{k} p^{k} W^{k}\left(v_{0}, x\right)$, which is well-defined thanks to Lemma 1.

In $\Gamma$, if $X(K) \neq \emptyset$, a nonrevealing equilibrium can be achieved as above, provided that player 2 chooses $x^{*} \in X$ to maximize $\sum_{k} p^{k} V^{k}(x)$ subject to $x \in X(K)$. If $X(K)=\emptyset$ and player 1's message is type-independent, condition (7) cannot be satisfied.

The next two propositions (which are established in Section 6.1) give us some foundations to study the equilibria of the limit game $\Gamma$ by making precise relationships between the latter and the equilibria without exit of $\Gamma\left(v_{0}\right)$.

Proposition 3. Let $(\sigma, \tau)$ be an equilibrium without exit in $\Gamma\left(v_{0}\right)$, for some $v_{0} \in \mathbb{R}$. Then $(\sigma, \tau)$ is an equilibrium without exit in $\Gamma\left(z_{0}\right)$ for every $z_{0} \in \mathbb{R}$ such that $z_{0} \leq v_{0}$ and is also an equilibrium in $\Gamma$, with the same interim expected utility as in $\Gamma\left(v_{0}\right)$ for both players.

In other words, if $\Gamma$ has no a equilibrium (which indeed may happen, see Section 4.3), then, whatever $v_{0} \in \mathbb{R}, \Gamma\left(v_{0}\right)$ has no equilibrium without exit, that is, all equilibria of $\Gamma\left(v_{0}\right)$ must involve non-participation of at least one type.

Proposition 4. Let $(\sigma, \tau)$ be an equilibrium in $\Gamma$. Then there exists $v_{0} \in \mathbb{R}$ such that, for every $z_{0} \leq v_{0},(\sigma, \tau)$ is an equilibrium without exit of $\Gamma\left(z_{0}\right)$, with the same interim expected utility as in $\Gamma$ for both players.

The previous properties are useful under a mechanism design perspective. Assume player 2 is a "principal" who cannot commit to a mechanism $\mu$ : $K \rightarrow X$ but receives a message from the agent (player 1 ) and then, makes a decision in $X$ subject to the agent's participation constraints. With this 
interpretation, which turns out to be an extreme case of Bester and Strausz (2001)'s model, an optimal mechanism amounts to an equilibrium of $\Gamma\left(v_{0}\right)$ which maximizes player 2's ex ante expected utility. At a given $v_{0}$, the equilibrium of $\Gamma\left(v_{0}\right)$ that is best for player 2 may involve the exit of some types of player 1 . By contrast, player 2's best equilibrium payoff in the limit game $\Gamma$, when it exists, is achieved at an equilibrium $\left(\sigma^{*}, \tau^{*}\right)$ without exit. Let us denote player 2's corresponding payoff as $v_{N E}^{*}$. By Proposition 4, if $v_{0}$ is small enough, $\left(\sigma^{*}, \tau^{*}\right)$ is an equilibrium without exit in $\Gamma\left(v_{0}\right)$, giving the same expected utility $v_{N E}^{*}$ to player 2. By Proposition $3, v_{N E}^{*}$ is the best equilibrium payoff player 2 can achieve at an equilibrium without exit in $\Gamma\left(v_{0}\right)$. Moreover, as we show in details in Section 6.1, if $v_{0}$ is sufficiently low, player 2 cannot expect an expected utility higher than $v_{N E}^{*}$ at an equilibrium of $\Gamma\left(v_{0}\right)$ with exit of some types. ${ }^{7}$ Summing up, if the limit game $\Gamma$ has an equilibrium, an optimal mechanism, when the principal's utility $v_{0}$ in case of exit is sufficiently low, can be found by maximizing player 2's utility over the equilibria of $\Gamma$, without worrying about the precise level $v_{0}$.

\section{Existence of a partitional equilibrium in $\Gamma$}

In this section, we focus on the game $\Gamma$. We identify various sufficient conditions for the existence of a partitional equilibrium in $\Gamma$, in which player 1 uses a pure strategy, namely a mapping $\sigma: K \rightarrow M$, to send his message to player 2. The strategy $\sigma$ then induces the partition $\left\{K_{m}, m \in \sigma(K)\right\}$ of $K$, with $K_{m}=\sigma^{-1}(m)=\{k \in K: \sigma(k)=m\}$. In this case, (4) and (5) become respectively:

$$
\text { For every } m \in M, P_{\sigma}(m)=\sum_{k \in K_{m}} p^{k}
$$

For every $k \in K$ and $m \in M$ s.t. $P_{\sigma}(m)>0, p_{m}^{k}(\sigma)=\frac{p^{k} I\left(k \in K_{m}\right)}{P_{\sigma}(m)}$.

As in Section 2, for player 2, we focus on strategies of the form $\tau: M \rightarrow$ $X$. At an equilibrium of $\Gamma$, given player 1's strategy $\sigma$ and the message $m$ he receives, player 2 updates his belief over $K$ into $p_{m}(\sigma)$. To avoid exit, we must have

\footnotetext{
${ }^{7}$ The observation that the principal's ex ante expected utility is maximized when all types of the agent participate is also made in Bester and Strausz (2001), footnote 8.
} 


$$
X\left(\operatorname{supp} p_{m}(\sigma)\right) \neq \emptyset
$$

Player 2's strategy $\tau$ is then a best response to $\sigma$ in $\Gamma$ if and only if

$$
\forall m \in M \text { s.t. } P_{\sigma}(m)>0, \tau(m) \in \arg \max _{x \in X\left(\operatorname{supp} p_{m}(\sigma)\right)} \sum_{k} p_{m}^{k}(\sigma) V^{k}(x) .
$$

We refer to these conditions as to constrained optimization.

Player 1's equilibrium conditions reduce to incentive compatibility conditions expressing that given player 2's strategy $\tau$, player 1 of type $k$ prefers to send $\sigma(k)$ than any other message $m$, namely,

$$
\left.U^{k}(\tau(\sigma(k)))\right) \geq U^{k}(\tau(m)) \text { for every } k \in K \text { and } m \in M \text {. }
$$

\subsection{Two types}

If only two types are possible, we show that either there is a decision giving both types at least their reservation utility or full revelation of information is credible and allows to avoid exit.

Proposition 5. Let us assume that $|K|=2$. Then $\Gamma$ has a partitional equilibrium.

Proof: If $X(1) \cap X(2) \neq \emptyset$, let

$$
x^{*} \in \arg \max _{x \in X(1) \cap X(2)}\left[p^{1} V^{1}(x)+p^{2} V^{2}(x)\right]
$$

and let $m^{*}$ be an arbitrary element of $M$. Then $\sigma(1)=\sigma(2)=m^{*}$ and $\tau(m)=x^{*}$ for every $m \in M$ defines a nonrevealing equilibrium of $\Gamma$.

Otherwise, if $X(1) \cap X(2)=\emptyset$, let

$$
x_{k} \in \arg \max _{x \in X(k)} V^{k}(x) \quad k=1,2
$$

and let $m_{1} \neq m_{2}$ be two distinct elements of $M$. Then $\sigma(k)=m_{k}, \tau\left(m_{k}\right)=$ $x_{k}, k=1,2$, defines a fully revealing equilibrium of $\Gamma$. Indeed, constrained optimization (11) holds by construction; to see that incentive compatibility (12) also holds, observe that $x_{k} \in X(k)$. Hence $x_{k} \notin X(\ell)$ for $\ell \neq k$, since $X(1) \cap X(2)=\emptyset$. In other words, $U^{\ell}\left(x_{k}\right)<u_{0}^{\ell} \leq U^{\ell}\left(x_{\ell}\right)$ for $\ell \neq k$. 


\subsection{Straightforward partitional equilibria}

In this section, we propose an easy generalization of Proposition 5 when the sender has an arbitrary number of types. Recalling the definition of $X(L)$ (see (1)), let us set

$$
\mathcal{T}=\{\emptyset \neq L \subseteq K: X(L) \neq \emptyset\}
$$

and define $\mathcal{T}^{*}$ as the set of maximal elements of $\mathcal{T}$ for set inclusion, namely,

$$
\mathcal{T}^{*}=\left\{L \in \mathcal{T}:\left[L^{\prime} \in \mathcal{T} \text { and } L \subseteq L^{\prime}\right] \Rightarrow L^{\prime}=L\right\} .
$$

We refer to $\mathcal{T}^{*}$ as to the "participation structure" of the game $\Gamma$.

Proposition 6. If the participation structure of $\Gamma$ is a partition of $K, \Gamma$ has a partitional equilibrium.

\section{Proof:}

Let $\mathcal{T}^{*}=\left\{K_{r}\right\}$. Consider the strategy of player 1 consisting of revealing the cell $K_{r}$ containing his type. Let $x_{r}^{*} \in X\left(K_{r}\right)$ be an optimal decision of player 2 when he learns that player 1's type belongs $K_{r}$, namely,

$$
x_{r}^{*} \in \arg \max _{x \in X\left(K_{r}\right)} \sum_{k \in K_{r}} \frac{p^{k}}{\sum_{j \in K_{r}} p^{j}} V^{k}(x) .
$$

Constrained optimization (11) holds by construction. Incentive compatibility (12) is also immediate, because if $k \in K_{r}, x_{r}^{*} \in X\left(K_{r}\right)$ while for $j \neq r$, $x_{j}^{*} \notin X\left(K_{r}\right)$.

\subsection{Decision in a real interval and monotonic utility function for the sender}

The next result holds in particular when player 2's decision can be interpreted as a probability distribution over two possible actions (i.e., $X=\Delta(A),|A|=$ 2 ) and the utility $U^{k}(x)$ of player 1 of type $k$ is expected utility with respect to $x$.

Proposition 7. Let us assume that the decision set $X$ is a real interval and every utility function $U^{k}, k \in K$, is monotonic over $X$. Then $\Gamma$ has a partitional equilibrium. 


\section{Proof:}

Let us take, without loss of generality, $X=[0,1]$; define

$$
\begin{aligned}
& K_{-}=\left\{k \in K: U^{k} \text { is weakly decreasing and not constant }\right\} \\
& K_{+}=\left\{k \in K: U^{k} \text { is weakly increasing or constant }\right\}
\end{aligned}
$$

We can find $x_{0}^{k}, k \in K$, such that if $k \in K_{-}, U^{k}(x) \geq u_{0}^{k} \Leftrightarrow x \leq x_{0}^{k}$ and if $k \in K_{+}, U^{k}(x) \geq u_{0}^{k} \Leftrightarrow x \geq x_{0}^{k}$. We define next

$$
\begin{array}{llll}
x_{-}=\min _{k \in K_{-}} x_{0}^{k} & \text { if } K_{-} \neq \emptyset ; & x_{-}=1 & \text { if } K_{-}=\emptyset . \\
x_{+}=\max _{k \in K_{+}} x_{0}^{k} & \text { if } K_{+} \neq \emptyset ; & x_{+}=0 & \text { if } K_{+}=\emptyset .
\end{array}
$$

If $x_{+} \leq x_{-}$(in particular, if $K_{-}$or $K_{+}=\emptyset$ ), let

$$
x^{*} \in \arg \max _{\left[x_{+}, x_{-}\right]} \sum_{k \in K} p^{k} V^{k}(x)
$$

and let $m^{*}$ be an arbitrary element of $M$. Then $\sigma(k)=m^{*}$ for every $k \in K$ and $\tau(m)=x^{*}$ for every $m \in M$ defines a nonrevealing equilibrium of $\Gamma$.

If $x_{+}>x_{-}$, let $m_{-}^{*} \neq m_{+}^{*}$ be two distinct elements of $M$. Take $\sigma(k)=$ $m_{-}^{*}$ if $k \in K_{-}, \sigma(k)=m_{+}^{*}$ if $k \in K_{+}$, namely, $\sigma$ induces the partition $\left\{K_{-}, K_{+}\right\}$. Player 2's corresponding posterior probability distribution on $K$ can be computed as in (9):

$$
p_{m_{-}^{*}}^{k}=\frac{p^{k} I\left(k \in K_{-}\right)}{\sum_{j \in K_{-}} p^{j}} \text { and } p_{m_{+}^{*}}^{k}=\frac{p^{k} I\left(k \in K_{+}\right)}{\sum_{j \in K_{+}} p^{j}} .
$$

Let then

$$
x_{-}^{*} \in \arg \max _{\left[0, x_{-}\right]} \sum_{k \in K_{-}} p_{m_{-}^{*}}^{k} V^{k}(x) \text { and } x_{+}^{*} \in \arg \max _{\left[x_{+}, 1\right]} \sum_{k \in K_{+}} p_{m_{+}^{*}}^{k} V^{k}(x) .
$$

Constrained optimization (11) holds by construction. There remains to check incentive compatibility (12). Observe that $x_{-}^{*}<x_{+}^{*}$; for $k \in K_{-}, U^{k}$ is decreasing, hence $U^{k}\left(x_{-}^{*}\right) \geq U^{k}\left(x_{+}^{*}\right)$. Similarly for $k \in K_{+}, U^{k}$ is increasing so that $U^{k}\left(x_{-}^{*}\right) \leq U^{k}\left(x_{+}^{*}\right)$. 


\subsection{Type-independent utility function for the receiver}

In this section, we assume that, when the sender accepts the receiver's proposal, the utility function of the receiver does not depend on the sender's type, namely, that $V^{k}(x)=V(x)$ for every $k$ and $x$. This assumption is sometimes referred to as "private values" or "known-own payoff." Matthews (1989) formulates it in the context of a game of information transmission with sender's approval.

Theorem 8. Let us assume that player 2's utility function does not depend on player 1's type, namely, that there exists a continuous function $V: X \rightarrow \mathbb{R}$ such that $V^{k}=V$ for every $k \in K$. Then $\Gamma$ has a partitional equilibrium.

The proof consists of an algorithm, which constructs a partitional equilibrium that is as revealing as possible, given the incentive compatibility constraints to be fulfilled. More precisely, the initial candidate is the fully revealing equilibrium. Imagine that type $k$ would envy type $\ell$ if one tried to implement fully revealing strategies, while type $\ell$ would not envy any type. By merging type $\ell$ and type $k$, one reduces the incentives problem. A key property is that, if player 2's utility function is independent of player 1's type, then player 2's optimal decision $x_{\ell}$ when facing type $\ell$ remains optimal when facing type $\ell$ or type $k$. Before making use of it, we first show, by relying on the same kind of argument, that the envy relation cannot have any cycle.

\section{Proof:}

Let us fix, for every $k \in K$,

$$
x_{k} \in \arg \max _{x \in X(k)} V(x) .
$$

The existence of such $x_{k}$ 's is guaranteed by our assumptions. If the previous optimization problem has several solutions, we take $x_{k}$ to maximize $U^{k}(x)$.

For every pair of types $j, k \in K$, we say that "type $k$ envies type $j$ " and write $k \mathcal{R} j$ - if $U^{k}\left(x_{j}\right)>U^{k}\left(x_{k}\right)$. An immediate property is that

$$
\text { for every } j, k \in K, k \mathcal{R} j \Rightarrow V\left(x_{k}\right)>V\left(x_{j}\right) \text {. }
$$

To show this, observe that, by definition, $x_{k} \in X(k)$, i.e., $U^{k}\left(x_{k}\right) \geq u_{0}^{k}$. Hence, if $k \mathcal{R} j$, we must have $U^{k}\left(x_{j}\right)>u_{0}^{k}$, which implies $x_{j} \in X(k)$ (so that 
$\left.x_{j} \in X(j) \cap X(k)\right)$ and $V\left(x_{k}\right) \geq V\left(x_{j}\right)$. But $V\left(x_{k}\right)=V\left(x_{j}\right)$ cannot arise, because $U^{k}\left(x_{j}\right)>U^{k}\left(x_{k}\right)$ and, in case of multiple solutions to $\max _{x \in X(k)} V(x)$, we choose $x_{k}$ to maximize $U^{k}(x)$.

The previous property implies that the envy relation $\mathcal{R}$ has no cycle.

We will gradually construct a subset $L \subseteq K$ of leader types which do not envy any other type in $L$ and a subset $F=K \backslash L$ of follower types which envy a type in $L$.

We start with $L=F=\emptyset$. Let us denote as $\alpha_{1}<\cdots<\alpha_{n}$ the distinct values among $V\left(x_{k}\right), k \in K$. Necessarily, $n \leq|K|$. Define then

$$
K_{j}=\left\{k \in K: V\left(x_{k}\right)=\alpha_{j}\right\} \quad j=1, \ldots, n .
$$

Step 1 Consider every type $k \in K_{1}: V\left(x_{k}\right)=\alpha_{1}$ is strictly below any other $\alpha_{j}$. By (15), type $k$ cannot envy any other type. We modify $L$ into $L=K_{1}$, while $F$ does not change $(F=\emptyset)$.

Step 2 Consider every type $k \in K_{2}$. If $k$ does not envy any type, put $k$ in

$L$. Otherwise, again by (15), $k$ can only envy a type in $L$ (as defined at the end of step 1, namely, $K_{1}$ ), put $k$ in $F$.

Step $j$ Let $L$ and $F$ be the sets of leaders and followers constructed so far. $L \cup F=K_{1} \cup \cdots \cup K_{j-1}$ so that by (15) and (16), types in $L \cup F$ cannot envy types in $K_{j}$. Consider every such type $k \in K_{j}$. If $k$ envies a type in $L$, put $k$ in $F$. Otherwise, put $k$ in $L$. $L$ and $F$ are thus updated at the end of step $j$.

Step $\boldsymbol{n}$ Proceed as for step $j$. Deduce the final sets of leaders and followers.

For instance, if $|K|=3$ and $\mathcal{R}$ is fully described by $3 \mathcal{R} 2$ and $2 \mathcal{R} 1$, the previous construction results in $K_{1}=\{1\}, K_{2}=\{2\}, K_{3}=\{3\}, L=\{1,3\}$.

Using the $x_{k}$ 's defined by (14) and the set $L$, we construct an equilibrium $(\sigma, \tau)$ of $\Gamma$. For simplicity, we rename the messages in $M$ so that $L \subseteq M$. Player 1's strategy is such that $\sigma(K)=L$. More precisely, $\sigma: K \rightarrow L$ is defined by

$$
\begin{aligned}
& \sigma(k)=k \quad \text { if } k \in L \\
& \arg \max _{j \in L, k \mathcal{R} j} U^{k}\left(x_{j}\right) \quad \text { if } k \in K \backslash L \text {. }
\end{aligned}
$$


In other words, leader types announce themselves, while non leader types report the leader type they most envy. Player 2's strategy is defined by $\tau$ : $L \rightarrow X: \tau(\ell)=x_{\ell}$, with $x_{\ell}$ defined by (14).

Incentive compatibility (12) follows from the fact that player 2's strategy $\tau$ restricts his decisions to the subset $\left\{x_{\ell}, \ell \in L\right\}$. Hence types in $L$, who cannot envy any other type in $L$, are truthful. Types in $K \backslash L$ behave as well as they can given the player 2's restricted decision set.

If player 1 follows $\sigma$, then, given message $\ell \in L$, player 2 deduces that player 1's type $k \in \sigma^{-1}(\ell)$. The set $\sigma^{-1}(\ell)$ contains $\ell, x_{\ell} \in X(\ell)$ by (14) and all other types in $\sigma^{-1}(\ell)$ envy $\ell$, so that $x_{\ell} \in \bigcap_{k \in \sigma^{-1}(\ell)} X(k)$. Since $x_{\ell}$ is a maximizer of $V(x)$ over $X(\ell)$, it is also a maximizer of $V(x)$ over $\bigcap_{k \in \sigma^{-1}(\ell)} X(k)$.

\section{Remarks:}

- A main feature of the proof of Theorem 8 is that, in the partitional equilibrium that is constructed, the receiver makes a decision in a subset of $\left\{x_{k}, k \in K\right\}$ where $x_{k}$ is the optimal decision he would make if he were sure to face type $k$. The receiver's private values guarantee that if type $k$ envies type $\ell$, then $x_{\ell}$, the receiver's optimal choice when he faces type $\ell$ for sure (i.e., under the constraint $x \in X(\ell)$ ), is still optimal when he faces type $k$ or type $\ell$ (i.e., under the constraint $x \in X(\ell) \cap X(k)$ ). This property may no longer hold when player 2's utility is type-dependent.

- Theorem 8 does not depend on the underlying utility representation: the result holds if the receiver's von Neumann-Morgenstern preferences over $X$ given type $k$ are equivalent for every $k \in K$.

\section{Examples (including a counter-example)}

\subsection{Partitional equilibrium}

Let the informed player have three possible types, i.e., $K=\{1,2,3\}$ and let the uninformed player's decision set be

$$
X=\left\{\left(x_{a}, x_{b}\right): x_{a} \geq 0, x_{b} \geq 0, x_{a}+x_{b} \leq 100\right\} .
$$

Let the utility function and reservation utility of the informed player be 


$$
\begin{array}{cc}
U^{1}(x)=x_{a}-x_{b} & u_{0}^{1}=30 \\
U^{2}(x)=x_{b}-x_{a} & u_{0}^{2}=40 \\
U^{3}(x)=x_{a}+2 x_{b} & u_{0}^{3}=20 .
\end{array}
$$

Let the uninformed player's utility function be type-independent:

$$
V^{k}(x)=V(x)=-\left(x_{a}+x_{b}\right), k=1,2,3 .
$$

There are two goods, $a$ and $b, X$ accounts for the decision-maker's resource constraints. Type 1 likes good $a$, dislikes good $b$; type 2 has symmetric preferences; type 3 likes both goods, and likes good $b$ more than good $a$.

As a possible interpretation, player 1 is a kidnapper who can have political motivations (type 1), just look for a monetary ransom (type 2) or be opportunistic (type 3). Good $a$ stands for political prisoners who can be released while good $b$ stands for money. If player 1 does not accept player 2's offer, the hostage is killed, leading to an invaluable loss for player 2 .

Recalling (1) and using " $\mathrm{Co}$ " for convex hull, we have here

$$
\begin{aligned}
X(\{1\}) & =X(\{1,3\})=C o\{(30,0),(100,0),(65,35)\}, \\
X(\{2\}) & =X(\{2,3\})=\operatorname{Co}\{(0,40),(0,100),(30,70)\}, \\
X(\{3\}) & =C o\{(20,0),(100,0),(0,100),(0,10)\}, \\
X(\{1,2\}) & =X(\{1,2,3\})=\emptyset .
\end{aligned}
$$

Assume first that player 1's type $k$ is known, namely, that $p^{k}=1$. Let then $x_{k}^{*}$ be the uninformed player's optimal decision (in $X$ ) when he faces type $k$ :

$$
x_{1}^{*}=(30,0), x_{2}^{*}=(0,40), x_{3}^{*}=(0,10) .
$$

Suppose next that only two types are possible. If $p^{1}=0$, given that $X(\{2,3\}) \neq \emptyset$, there is a nonrevealing equilibrium, $x_{2}^{*}=(0,40)$. Similarly for $p^{2}=0$, with $x_{1}^{*}=(30,0)$. If $p^{3}=0$, there is no way to satisfy type 1 and type 2 at the same time. But there is a completely revealing equilibrium: $x_{1}^{*}=(30,0)$ to type $1, x_{2}^{*}=(0,40)$ to type 2 is incentive compatible. This illustrates Proposition 5.

Let the three types be possible, namely $p^{k}>0$ for $k=1,2,3$. There is no nonrevealing equilibrium, since $X(\{1,2,3\})=\emptyset$. There is no completely revealing equilibrium either: (17) implies that type 3 would pretend to be type 2 (type 3 envies type 1 and type 2 even more). 
As expected from Theorem 8, there exists a partitional equilibrium. The informed player is invited to report whether his type is 1 or not. If he reports type 1 , the uninformed player proposes $x_{1}^{*}=(30,0)$. If the informed player reports that his type is not 1 , the uninformed player proposes

$$
\arg \min _{x \in X(\{2,3\})}\left(x_{a}+x_{b}\right)=x_{2}^{*}=(0,40) .
$$

As in the proof of Theorem 8, incentive compatibility is ensured by the fact that the decision proposed to type 3 is the one he most envies among $x_{1}^{*}$ and $x_{2}^{*}$.

\subsection{Mixed equilibrium}

Let us modify the uninformed player's utility function in the previous example, to make it depend on the informed player's type:

$$
\begin{aligned}
V^{1}(x) & =\frac{x_{a}}{3}, \\
V^{2}(x) & =\frac{x_{b}}{3}, \\
V^{3}(x) & =-\left(x_{a}+x_{b}\right) .
\end{aligned}
$$

A possible interpretation is that the decision-maker is happy to pay when the kidnapper has sharp preferences.

Let as above $x_{k}^{*}$ denote the uninformed player's optimal decision (in $X$ ) when he faces type $k$; we have now

$$
x_{1}^{*}=(100,0), x_{2}^{*}=(0,100), x_{3}^{*}=(0,10) .
$$

Let us take $p=\left(\frac{1}{3}, \frac{1}{3}, \frac{1}{3}\right)$. There is no nonrevealing equilibrium, since $X(\{1,2,3\})=\emptyset$. There is no completely revealing equilibrium: given (18), type 3 would pretend to be type 2 . More generally, there is no partitional equilibrium. Given the above description of the sets $X(L)$, two possible partitions must still be considered: $\{\{1\},\{2,3\}\}$ and $\{\{1,3\},\{2\}\}$.

$\{\{1\},\{2,3\}\}$ : if the uninformed player believes he faces type 1 (posterior $(1,0,0))$, his optimal choice is $x_{1}^{*}=(100,0)$; if he believes he faces type 2 or type 3 (posterior $\left(0, \frac{1}{2}, \frac{1}{2}\right)$ ), his optimal choice is

$$
x_{23}^{*}=\arg \min _{x \in X(\{2,3\})}\left[x_{a}+\frac{2}{3} x_{b}\right]=(0,40) .
$$


This cannot be incentive compatible for type 3 :

$$
100=U^{3}\left(x_{1}^{*}\right)>U^{3}\left(x_{23}^{*}\right)=80 .
$$

$\{\{1,3\},\{2\}\}$ : if the uninformed player believes he faces type 2 (posterior $(0,1,0))$, his optimal choice is $x_{2}^{*}=(0,100)$; if he believes he faces type 1 or type 3 (posterior $\left(\frac{1}{2}, 0, \frac{1}{2}\right)$ ), his optimal choice is

$$
x_{13}^{*}=\arg \min _{x \in X(\{1,3\})}\left[\frac{2}{3} x_{a}+x_{b}\right]=(100,0) .
$$

Again, this cannot be incentive compatible for type 3 :

$$
200=U^{3}\left(x_{2}^{*}\right)>U^{3}\left(x_{13}^{*}\right)=100 .
$$

This illustrates that Theorem 8 does not extend to the case where player 2's utility function depends on player 1's type.

Let us show that if player 1 uses a mixed strategy, a partially revealing equilibrium exists in this example: type 1 reports that his type belongs to $\{1,3\}$, type 2 reports that his type belongs to $\{2,3\}$, type 3 reports that his type belongs to $\{1,3\}$ (resp., $\{2,3\}$ ) with probability $\frac{1}{3}$ (resp., $\frac{2}{3}$ ). If the informed player follows this reporting strategy, the uninformed player's posterior upon receiving $\{1,3\}$ is $\left(\frac{3}{4}, 0, \frac{1}{4}\right)$ while upon receiving $\{2,3\}$, it is $\left(0, \frac{3}{5}, \frac{2}{5}\right)$. Given $\{1,3\}$, the uninformed player's problem reduces to $\min _{x \in X(\{1,3\})} x_{b}$. Every $x=\left(x_{a}, 0\right)$ with $x_{a} \in[30,100]$ is optimal. Let us take $x_{13}^{*}=(80,0)$. Given $\{2,3\}$, the uninformed player's optimal choice is

$$
x_{23}^{*}=\arg \min _{x \in X(\{2,3\})}\left[2 x_{a}+x_{b}\right]=(0,40) .
$$

There remains to check incentive compatibility. Type 1 prefers $x_{13}^{*}=(80,0)$ to $x_{23}^{*}=(0,40)$, and vice-versa for type 2 . Type 3 must be indifferent between sending $\{1,3\}$ or $\{2,3\}$, because he must randomize between these two outcomes. Indeed we have $U^{3}\left(x_{13}^{*}\right)=U^{3}\left(x_{23}^{*}\right)=80$. Proposition 9 in Section 5 states that the previous construction can be generalized.

\subsection{No equilibrium at all}

In the following example, none of the existence results of Section 3 can be applied. We will show that there is no equilibrium, even if player 1 makes use 
of a mixed strategy. The game is described by: $K \mid=3, X=\Delta(A)$, where $A=\{a, b, c\}, u_{0}^{k}=0, k=1,2,3$. The following payoff matrices describe $\left(U^{k}(\alpha), V^{k}(\alpha)\right)$ for every $\alpha \in A$ :

$$
\begin{array}{cccc} 
& a & b & c \\
k=1 & 0,2 & -2,0 & 1,1 \\
k=2 & 1,1 & 0,2 & -2,0 \\
k=3 & -2,0 & 1,1 & 0,2
\end{array}
$$

The utility functions over $X=\Delta(A)$ are obtained as expected utilities with respect to $x=\left(x_{a}, x_{b}, x_{c}\right)$.

If player 2 knows that he faces type $k$ (i.e., $p^{k}=1$ ), he gets his first best by choosing $a$ if $k=1, b$ if $k=2, c$ if $k=3$. But if $p^{k}>0$ for every $k$, there is no nonrevealing equilibrium $\left(\sum_{k} U^{k}(x)<0\right.$ for every $\left.x \in \Delta(A)\right)$ and no fully revealing equilibrium (incentive compatibility is violated).

Looking for a partially revealing equilibrium, we first check that there is a unique, nonrevealing equilibrium, as soon as only two types are possible. Take, e.g., $p^{3}=0$. Then

$$
\begin{aligned}
X(\{1,2\}) & =\left\{x \in X:-2 x_{b}+x_{c} \geq 0 \text { and } x_{a}-2 x_{c} \geq 0\right\} \\
& =\operatorname{Co}\left\{(1,0,0),\left(\frac{2}{3}, 0, \frac{1}{3}\right),\left(\frac{4}{7}, \frac{1}{7}, \frac{2}{7}\right)\right\}
\end{aligned}
$$

and player 2's optimization problem is:

$$
\max p^{1}\left(2 x_{a}+x_{c}\right)+p^{2}\left(x_{a}+2 x_{b}\right) \text { s.t. } x \in X(\{1,2\}) .
$$

For every $p$ such that $p^{1}>0$ and $p^{2}>0$, the unique solution is achieved at $x=(1,0,0)$, namely, action $a$ with probability 1 . Similarly, action $b$ is the only solution if $p^{2}>0$ and $p^{3}>0$, action $c$ is the only solution if $p^{1}>0$ and $p^{3}>0$.

Let us start with $p$ such that $p^{k}>0$ for every $k$. By sending his message according to a mixed strategy $\sigma$, player 1 "splits" the prior belief $p$ into posteriors $p_{m}(\sigma)$ such that $\sum_{m} P_{\sigma}(m) p_{m}(\sigma)=p$ (see $(5)$ ). Taking account 
of incentive compatibility, $p$ cannot be split (only) extreme points, because there is no fully revealing equilibrium. At least one of the posteriors $p_{m}$ must be on an edge, say $p_{m}^{3}=0$, so that $\tau(m)=a$. There should be another posterior $p_{m^{\prime}}$ with $p_{m^{\prime}}^{3}>0$, with $\tau\left(m^{\prime}\right)=b$ or $c$. To achieve the posteriors $p_{m}$ and $p_{m^{\prime}}$, message $m$ must be sent with positive probability by types 1 and 2 , while message $m^{\prime}$ must be sent with positive probability by at least type 3. If $\tau\left(m^{\prime}\right)=b$, type 2 strictly prefers $m^{\prime}$ to $m$. If $x\left(m^{\prime}\right)=c$, type 1 strictly prefers $m^{\prime}$ to $m$. Hence there is no incentive compatible splitting and thus no equilibrium at all, even if player 1 can use a mixed strategy.

A mediated equilibrium, in which information transmission is monitored by a mediator, can nevertheless be achieved in the previous example. Consider the following three lotteries over $A$ : $\delta^{1}=\left(\frac{1}{2}, 0, \frac{1}{2}\right), \delta^{2}=\left(\frac{1}{2}, \frac{1}{2}, 0\right)$, $\delta^{3}=\left(0, \frac{1}{2}, \frac{1}{2}\right)$. Assume that, instead of selecting a message by himself, player 1 can just choose among these three lotteries. If player 1 expects player 2 to pick the action selected by the lottery, player 1 prefers $\delta^{k}$ over the other two lotteries when his type is $k$. Similarly, player 2 is happy to choose the action recommended by the lottery if he believes that player 1 reveals his type truthfully to the mediator. This procedure will be generalized in the next section.

\section{$5 \quad$ Equilibrium in the case of three types}

In this section, we propose a thorough analysis of the equilibria of $\Gamma$ when player 1 has three possible types $(|K|=3)$. Recall that $\mathcal{T}^{*}$ denotes the participation structure of $\Gamma$ (see (1) and (13)).

When $|K|=3$, there are three typical cases:

1. $\mathcal{T}^{*}$ is a partition of $K$.

2. $\mathcal{T}^{*}=\{\{1,2\},\{1,3\},\{2,3\}\}$.

3. $\mathcal{T}^{*}=\{\{1,3\},\{2,3\}\}$.

In case 1 , by Proposition $6, \Gamma$ has a partitional equilibrium. Case 2 means that player 2 is able to obtain the approval of every pair of types but cannot ensure the participation of the three types simultaneously. In this case, as illustrated in Section 4.3, $\Gamma$ may have no mixed equilibrium. We will show 
below that $\Gamma$ always has a mediated equilibrium. In case 3 , which has been illustrated in Section 4.2., player 2 can only guarantee the approval of two pairs of types. We will establish that under further assumptions, $\Gamma$ always has then a mixed (possibly not pure) equilibrium.

\section{$5.1 \mathcal{T}^{*}=\{\{1,2\},\{1,3\},\{2,3\}\}$}

Let us enrich the description of $\Gamma$ by adding a mediator who invites player 1 to report a type (in $K$ ) and then selects a decision (in $X$ ) that he recommends player 2 . At a mediated equilibrium, player 1 truthfully reveals his type to the mediator, player 2 proposes to player 1 the decision $x$ that is recommended by the mediator and finally, player 1 of type $k$ accepts player 2's proposal $x^{\prime}$ provided that $U^{k}\left(x^{\prime}\right) \geq u_{0}^{k}$.

Let us construct a mediated equilibrium in $\Gamma$ when $K=\{1,2,3\}$ and $\mathcal{T}^{*}=\{\{1,2\},\{1,3\},\{2,3\}\}$. Recall that $p^{k}>0$ for every $k$. For every pair $(j, k)$ of types, let $x_{j k}^{*} \in X(\{j, k\})$ be an optimal decision for player 2 when he learns that player 1 is of type $j$ or $k$, namely,

$$
x_{j k}^{*} \in \arg \max \left[\frac{p^{j}}{p^{j}+p^{k}} V^{j}(x)+\frac{p^{k}}{p^{j}+p^{k}} V^{k}(x)\right] .
$$

Consider the following mediator: for every $k=1,2,3$, if player 1 reports type $k$, he selects $x_{i k}^{*}$ or $x_{j k}^{*}, i, j \neq k, i \neq j$, with equal probability $\frac{1}{2}$ and recommends it to player 2 . If player 1 reports his type $k$ truthfully, player 2 learns, with equal probability, that player 1's type is in $\{i, k\}$ or in $\{j, k\}$ for $i, j \neq k, i \neq j$. Condition (19) guarantees that player 2 follows the mediator's recommendation.

For player 1 , let us consider $k=1$. By reporting his type truthfully, player 1 obtains

$$
\frac{1}{2} U^{1}\left(x_{12}^{*}\right)+\frac{1}{2} U^{1}\left(x_{13}^{*}\right) .
$$

If he lies by, say, pretending to be of type 2 , he obtains ${ }^{8}$

$$
U_{2}^{1}=\frac{1}{2} \max \left\{U^{1}\left(x_{12}^{*}\right), u_{0}^{1}\right\}+\frac{1}{2} \max \left\{U^{1}\left(x_{23}^{*}\right), u_{0}^{1}\right\} .
$$

${ }^{8}$ Player 1's incentive compatibility condition reflects the fact that this player can veto player 2's offer, namely, can lie about his type and/or reject player 2's proposal. This is a "veto-incentive compatibility condition" (see, e.g., Forges (1999)), which implies posterior individual rationality. 
By construction, $x_{12}^{*} \in X(\{1,2\})$ and $x_{13}^{*} \in X(\{1,3\})$. Hence $U^{1}\left(x_{12}^{*}\right) \geq u_{0}^{1}$ and $U^{1}\left(x_{13}^{*}\right) \geq u_{0}^{1}$. But $x_{23}^{*} \notin X(1)$ because $\{2,3\}$ is maximal. Hence, $U^{1}\left(x_{23}^{*}\right)<u_{0}^{1}$.

$$
U_{2}^{1}=\frac{1}{2} U^{1}\left(x_{12}^{*}\right)+\frac{1}{2} u_{0}^{1} \leq \frac{1}{2} U^{1}\left(x_{12}^{*}\right)+\frac{1}{2} U^{1}\left(x_{13}^{*}\right) .
$$

The other incentive compatibility conditions of player 1 can be checked in a symmetric way.

\section{$5.2 \mathcal{T}^{*}=\{\{1,3\},\{2,3\}\}$}

Proposition 9. Let us assume that $K=\{1,2,3\}$, the participation structure $\mathcal{T}^{*}=\{\{1,3\},\{2,3\}\}$, the decision set $X$ is compact and convex and the utility functions $U^{k}$ and $V^{k}, k \in K$, are affine. Then $\Gamma$ has a partially revealing equilibrium, in which player 1 uses a mixed, possibly not pure, strategy.

Proof: See Section 6.2. We establish that there must exist an equilibrium in which type 1 reports that his type belongs to $\{1,3\}$, type 2 reports that his type belongs to $\{2,3\}$ and type 3 reports that his type belongs to $\{1,3\}$ (resp., $\{2,3\}$ ) with some probability $\delta \in(0,1)$ (resp., $1-\delta$ ). Incentive compatibility requires that type 3 be indifferent between reporting $\{1,3\}$ or $\{2,3\}$. Such an equilibrium is shown to be the only possible one in the example of Section 4.2 .

\section{Appendix}

\subsection{Proof of Propositions 3 and 4}

For the sake of completeness, we first explicitly recall the conditions to be satisfied by an equilibrium of $\Gamma\left(v_{0}\right)$ whether they involve exit of some types on equilibrium path or not.

Let us fix a pair of strategies $\sigma: K \rightarrow \Delta(M)$ and $\tau: M \rightarrow X$. Player 1's equilibrium conditions can be written as

$$
U_{+}^{k}(\tau(m)) \geq U_{+}^{k}\left(\tau\left(m^{\prime}\right)\right) \forall k \in K, \forall m \in M \text { s.t. } \sigma(m \mid k)>0, \forall m^{\prime} \in M .
$$

Player 2's equilibrium conditions can be written as

$\sum_{k} p_{m}^{k}(\sigma) W^{k}\left(v_{0}, \tau(m)\right) \geq \sum_{k} p_{m}^{k}(\sigma) W^{k}\left(v_{0}, x\right) \forall m \in M$ s.t. $P_{\sigma}(m)>0, \forall x \in X$. 
We deduce that the necessary and sufficient conditions for $(\sigma, \tau)$ to be an equilibrium without exit are:

For player 1:

$$
U^{k}(\tau(m)) \geq U^{k}\left(\tau\left(m^{\prime}\right)\right) \forall k \in K, \forall m \in M \text { s.t. } \sigma(m \mid k)>0, \forall m^{\prime} \in M,
$$

implying that

$$
U^{k}(\tau(m))=U^{k}\left(\tau\left(m^{\prime}\right)\right) \forall m, m^{\prime} \in M \text { s.t. } \sigma(m \mid k)>0 \text { and } \sigma\left(m^{\prime} \mid k\right)>0 .
$$

For player 2:

$$
\tau(m) \in\left[\arg \max _{x \in X} \sum_{k} p_{m}^{k}(\sigma) W^{k}\left(v_{0}, x\right)\right] \cap X\left(\operatorname{supp} p_{m}(\sigma)\right),
$$

implying constrained optimization, namely (11).

Recall that by definition, an equilibrium of $\Gamma$ cannot involve exit, so that the conditions for $(\sigma, \tau)$ to be an equilibrium in $\Gamma$ are thus just (21) and (11).

Remarks on (21):

- As a refinement of subgame perfect equilibrium, Matthews (1989) strengthens (20) to (21) in the case of equilibria which typically involve exit on path.

- For an equilibrium without exit, player 1's equilibrium conditions take the simple form (21) because player 2's strategy $\tau$ is pure. In an equilibrium without exit, for every $k \in K$, player 1's equilibrium strategy consists of sending $m$, selected with probability $\sigma(m \mid k)$, and then accept player 2's proposal, namely, $\tau(m)$, which is fully anticipated at the time to choose $m$. A deviation consists of sending $m^{\prime} \in M$ (possibly such that $\left.\sigma\left(m^{\prime} \mid k\right)=0\right)$ and then, accept or reject player 2's proposal $\tau\left(m^{\prime}\right)$. Player 1's equilibrium conditions thus take the form $U^{k}(\tau(m)) \geq \max \left\{U^{k}\left(\tau\left(m^{\prime}\right)\right), u_{0}^{k}\right\} \forall k \in K, \forall m \in M$ s.t. $\sigma(m \mid k)>0, \forall m^{\prime} \in M$.

These are equivalent to (21), since (6) holds at an equilibrium without exit. If player 2's strategy $\tau$ were mixed $(\tau: M \rightarrow \Delta(X))$, we would have to write ${ }^{9}$

$$
\left.\sum_{x} \tau(x \mid m) U^{k}(x) \geq \sum_{x} \tau\left(x \mid m^{\prime}\right) \max \left\{U^{k}(x)\right), u_{0}^{k}\right\} .
$$

\footnotetext{
${ }^{9}$ The expression is similar to "veto-incentive compatibility" (see, e.g., Forges (1999)) and implies posterior individual rationality.
} 
Proposition 3. Let $(\sigma, \tau)$ be an equilibrium without exit in $\Gamma\left(v_{0}\right)$, for some $v_{0} \in \mathbb{R}$. Then $(\sigma, \tau)$ is an equilibrium without exit in $\Gamma\left(z_{0}\right)$ for every $z_{0} \in R$ such that $z_{0} \leq v_{0}$ and is also an equilibrium in $\Gamma$, with the same interim expected utility as in $\Gamma\left(v_{0}\right)$ for both players.

Proof: If $(\sigma, \tau)$ satisfies $(22)$ in $\Gamma\left(v_{0}\right)$, the same holds in $\Gamma\left(z_{0}\right)$ since for every $x \in X$,

$$
\sum_{k} p_{m}^{k}(\sigma) W^{k}\left(z_{0}, x\right) \leq \sum_{k} p_{m}^{k}(\sigma) W^{k}\left(v_{0}, x\right)
$$

and for every $x \in X\left(\operatorname{supp} p_{m}(\sigma)\right)$,

$$
\sum_{k} p_{m}^{k}(\sigma) W^{k}\left(z_{0}, x\right)=\sum_{k} p_{m}^{k}(\sigma) W^{k}\left(v_{0}, x\right)=\sum_{k} p_{m}^{k}(\sigma) V^{k}(x) .
$$

Furthermore, (11) must hold and player 1's equilibrium conditions (21) are the same in $\Gamma\left(v_{0}\right)$ and $\Gamma\left(z_{0}\right)$ or $\Gamma$ as long as player 2's strategy remains unchanged.

Proposition 4. Let $(\sigma, \tau)$ be an equilibrium in $\Gamma$. Then there exists $v_{0} \in \mathbb{R}$ such that, for every $z_{0} \leq v_{0},(\sigma, \tau)$ is an equilibrium without exit of $\Gamma\left(z_{0}\right)$, with the same interim expected utility as in $\Gamma$ for both players.

Proof: Let $(\sigma, \tau)$ be an equilibrium without exit in $\Gamma$. By definition, constrained optimization (11) holds, so that in particular $\tau(m) \in X\left(\operatorname{supp} p_{m}(\sigma)\right)$ for every $m$ such that $P_{\sigma}(m)>0$. Let us keep player 1's strategy, $\sigma$, fixed. Player 2's strategy $\tau$ remains a best reply to $\sigma$ in $\Gamma\left(v_{0}\right)$, with $v_{0} \leq$ $\min _{k \in K} \min _{x \in X} V^{k}(x)$, provided that $v_{0}$ is such that optimality of no exit holds, namely, recalling (22),

$$
\forall m \in M \text { s.t. } P_{\sigma}(m)>0, \sum_{k} p_{m}^{k}(\sigma) V^{k}(\tau(m)) \geq \max _{x \in X} \sum_{k} p_{m}^{k}(\sigma) W^{k}\left(v_{0}, x\right) .
$$

These can be viewed as finitely many inequalities over $v_{0}$, which have a solution in $\mathbb{R}$, since the RHS are well-defined, for every $v_{0} \leq \min _{k \in K} \min _{x \in X} V^{k}(x)$, by Lemma $1 .^{10}$ Hence there exists $v_{0}$ such that $(\sigma, \tau)$ is an equilibrium without exit of $\Gamma\left(v_{0}\right)$ and from Proposition 3 , in $\Gamma\left(z_{0}\right)$, for every $z_{0} \leq v_{0}$.

\footnotetext{
${ }^{10}$ The RHS of the inequalities can be rewritten as

$$
\max _{L \subsetneq \operatorname{supp}\left(p_{m}(\sigma)\right)} \max _{x \in X(L)}\left\{\sum_{k \in L} p_{m}^{k}(\sigma) V^{k}(x)+v_{0} \sum_{k \in \operatorname{supp}\left(p_{m}(\sigma)\right) \backslash L} p_{m}^{k}(\sigma)\right\} .
$$
}




\section{Application to a mechanism design problem}

In Section 3, we have established that, under various reasonable assumptions, $\Gamma$ has a partitional equilibrium $(\sigma, \tau)$, in which both $\sigma$ and $\tau$ are pure. In this case, we can easily compute the highest ex ante expected utility that player 2, interpreted here as the principal, can obtain at a partitional equilibrium of $\Gamma{ }^{11}$ As explained in Section 2, this application is inspired by a particular case of Bester and Strausz (2001)'s model.

Let us show that there exists $v_{0} \in \mathbb{R}$ such that, for every $z_{0} \leq v_{0}$, the highest ex ante expected utility player 2 can obtain at an arbitrary partitional equilibrium of $\Gamma\left(z_{0}\right)$ (which can involve exit or not) is the same as in $\Gamma$. More precisely, there exists $v_{0} \in \mathbb{R}$ such that, for every $z_{0} \leq v_{0}$, the best partitional equilibrium for player 2 in $\Gamma$ remains the best partitional equilibrium for player 2 in $\Gamma\left(z_{0}\right)$.

Let $v_{N E}^{*}$ be the highest ex ante expected utility player 2 can obtain at a partitional equilibrium of $\Gamma$. This number is well-defined if $\Gamma$ has a partitional equilibrium. Let $\left(\sigma^{*}, \tau^{*}\right)$ achieve the expected utility $v_{N E}^{*}$ for player 2 . Using Proposition 4 , there exists $v_{0}$ sufficiently small such that for every $z_{0} \leq v_{0}$, $\left(\sigma^{*}, \tau^{*}\right)$ is an equilibrium without exit of $\Gamma\left(z_{0}\right)$ with the same expected utility $v_{N E}^{*}$ for player 2. By Proposition 3, for every such $z_{0}$, there does not exist any equilibrium without exit giving a higher expected utility to player 2 (because such an equilibrium would still be an equilibrium of $\Gamma$, with the same expected utilities).

Let us consider the partitional equilibria $(\sigma, \tau)$ of $\Gamma\left(v_{0}\right)$ in which exit possibly occurs, i.e., in which the set

$$
K_{E}=\left\{k \in K: U^{k}(\tau \circ \sigma(k))<u_{0}^{k}\right\} \neq \emptyset,
$$

i.e., $p_{E}={ }_{\text {def }} \sum_{k \in K_{E}} p^{k}>0$. The highest expected utility player 2 can achieve at such an equilibrium is

$$
p_{E} v_{0}+\left(1-p_{E}\right) \bar{v}
$$

where

$$
\bar{v}=\max _{k \in K} \max _{x \in X} V^{k}(x) .
$$

If $v_{0}$ is such that, for every $p_{E}$ that can arise given the prior $p$,

$$
p_{E} v_{0}+\left(1-p_{E}\right) \bar{v} \leq v_{N E}^{*},
$$

\footnotetext{
${ }^{11}$ The number of pure equilibrium payoffs is finite, in the same way as the number of partitions of $K$. Hence as soon as there is a pure equilibrium in $\Gamma$, there is an equilibrium achieving the highest expected payoff for the receiver.
} 


$$
\text { namely, } v_{0} \leq \frac{1}{p_{E}}\left[v_{N E}^{*}-\left(1-p_{E}\right) \bar{v}\right]
$$

then $\left(\sigma^{*}, \tau^{*}\right)$ will guarantee the highest possible equilibrium utility to player 2 , in every game $\Gamma\left(z_{0}\right)$ with $z_{0} \leq v_{0}$.

Let $\underline{k}$ be the type with the smallest prior probability, namely, such that $p^{\underline{k}}=\min \left\{p^{1}, \cdots, p^{K}\right\}$. The inequality (23) will hold at every $p_{E}$ that can arise given the prior $p$ as soon as it holds at $p_{E}=p^{k}$ : we just have to require

$$
v_{0} \leq \frac{1}{p^{\underline{k}}}\left[v_{N E}^{*}-\left(1-p^{\underline{k}}\right) \bar{v}\right] .
$$

The previous result is quite intuitive: an upper bound on the receiver's expected utility at an equilibrium of $\Gamma\left(v_{0}\right)$ with exit is obtained when the receiver's proposal is rejected by only the least likely type, while the best possible utility is achieved at all the other types. If $v_{0}$ is sufficiently low, the best equilibrium utility for the receiver in $\Gamma\left(v_{0}\right)$ will be not be achieved at an equilibrium with exit, but rather at an equilibrium without exit, which is in turn is necessarily an equilibrium of $\Gamma$.

\subsection{Proof of Proposition 9}

Proposition 9. Let us assume that $K=\{1,2,3\}, \mathcal{T}^{*}=\{\{1,3\},\{2,3\}\}$ the decision set $X$ is compact and convex and the utility functions $U^{k}$ and $V^{k}, k \in K$, are affine. Then $\Gamma$ has a partially revealing equilibrium.

For simplicity we assume here that $u_{0}^{k}=0$ for each $k$. This is w.l.o.g. since we can translate the payoffs of each type of the sender. We start with preliminaries.

\subsubsection{Mappings and multi-valued mappings}

Define for each $p$ in $\Delta(K)$ :

$$
\begin{aligned}
& f(p)=\sup \left\{\sum_{k \in K} p^{k} V^{k}(x), x \in X(\operatorname{supp} p)\right\} \in \mathbb{R} \cup\{-\infty\}, \\
& Y(p)=\operatorname{Argmax} x_{x \in X(\operatorname{supp} p)} \sum_{k} p^{k} V^{k}(x) \subset X(\operatorname{supp} p), \text { and } \\
& \Phi(p)=\left\{\left(U^{k}(x)\right)_{k \in K}, x \in Y(p)\right\} \subset \mathbb{R}^{K} .
\end{aligned}
$$

The sets $Y(p)$ and $\Phi(p)$ are convex compact subsets of $\mathbb{R}$ and $\mathbb{R}^{K}$, respectively. If $X(\operatorname{supp} p) \neq \emptyset$, then $f(p) \in \mathbb{R}, Y(p) \neq \emptyset$ and $\Phi(p) \neq \emptyset$. For each $u \in \Phi(p)$, we have $u^{k} \geq 0$ for each $k \in \operatorname{supp} p$. At an equilibrium of $\Gamma$, if the belief of the receiver (after having received the message of the sender) 
is $p$, then he has to propose a decision in $Y(p)$, inducing a vector payoff in $\Phi(p)$ for the different types of player 1.

We will use in the sequel the following three lemmas (Lemma 12 is a simple mean-value theorem for correspondences).

Lemma 10. The mapping $f$ is u.s.c. and convex.

If $p_{n} \underset{n \rightarrow \infty}{\longrightarrow} p \in \Delta(K)$ with $\operatorname{supp} p_{n}=\operatorname{supp} p$ for each $n$, then $f\left(p_{n}\right) \underset{n \rightarrow \infty}{\longrightarrow}$ $f(p)$.

Proof: Suppose $p_{n} \underset{n \rightarrow \infty}{\longrightarrow} p$. Then for $n$ large enough, supp $p_{n} \supset \operatorname{supp} p$ so $X\left(\operatorname{supp} p_{n}\right) \subset X(\operatorname{supp} p)$. It follows that $\limsup _{n} f\left(p_{n}\right) \leq f(p)$. (whether $f(p)=-\infty$ or not)

If $\operatorname{supp} p_{n}=\operatorname{supp} p$ for each $n$, then $\left|f\left(p_{n}\right)-f(p)\right| \leq \sup _{x \in X} \sum_{k \in K} \mid p_{n}^{k}-$ $p^{k}|| V^{k}(x) \mid$, and $f\left(p_{n}\right) \underset{n \rightarrow \infty}{\longrightarrow} f(p)$.

If $p=\lambda p_{1}+(1-\lambda) p_{2}$ with $\lambda \in(0,1)$, then $\operatorname{supp} p=\operatorname{supp} p_{1} \cup \operatorname{supp} p_{2}$ and $X(\operatorname{supp} p)=X\left(\operatorname{supp} p_{1}\right) \cap X\left(\operatorname{supp} p_{2}\right)$. If $f(p)=-\infty$ then $\lambda f\left(p_{1}\right)+(1-$ d) $f\left(p_{2}\right) \geq f(p)$. Consider $x$ in $X(\operatorname{supp} p)$, we have $f\left(p_{1}\right) \geq \sum_{k \in K} p_{1}^{k} V^{k}(x)$ and $f\left(p_{2}\right) \geq \sum_{k \in K} p_{2}^{k} V^{k}(x)$, so $\lambda f\left(p_{1}\right)+(1-\lambda) f\left(p_{2}\right) \geq \sum_{k \in K} p^{k} V^{k}(x)$, and taking the supremum for $x$ in $X(\operatorname{supp} p)$ we get $\lambda f\left(p_{1}\right)+(1-\lambda) f\left(p_{2}\right) \geq f(p)$. Hence $f$ is convex.

Lemma 11. Consider a converging sequence $p_{n} \underset{n \rightarrow \infty}{\longrightarrow} p \in \Delta(K)$.

a) Assume $\limsup \sup _{n} f\left(p_{n}\right)=f(p)$. Then if $u_{n} \underset{n \rightarrow \infty}{\longrightarrow} u \in \mathbb{R}^{K}$, with $u_{n} \in$ $\Phi\left(p_{n}\right)$ for each $n$, we have $u \in \Phi(p)$,

b) Otherwise $\lim \sup _{n} f\left(p_{n}\right)<f(p)$. Then there exists $n_{0}$ such that for each $u \in \Phi(p)$ and $n \geq n_{0}$, one can find $k \in \operatorname{supp} p_{n} \backslash\{\operatorname{supp} p\}$ such that $u^{k}<0$.

Proof: a) Without loss of generality we assume that $f\left(p_{n}\right) \underset{n \rightarrow \infty}{\longrightarrow} f(p)$. Write $u_{n}=\left(U^{k}\left(x_{n}\right)\right)_{k \in K}$ with $x_{n}$ in $Y\left(p_{n}\right)$ for each $n$. By taking a converging subsequence we can assume that $x_{n}$ converges to some $x$ in $X$. Since $p_{n} \underset{n \rightarrow \infty}{\longrightarrow} p$, for $n$ large enough supp $p_{n} \supset \operatorname{supp} p$ so $x \in X(\operatorname{supp} p)$. And $\sum_{k \in K} p_{n}^{k} V^{k}\left(x_{n}\right)=f\left(p_{n}\right) \underset{n \rightarrow \infty}{\longrightarrow} f(p)$, so $\sum_{k \in K} p^{k} V^{k}(x)=f(p)$. Then $x$ belongs to $Y(p)$, and $u \in \Phi(p)$.

b) Assume that $\limsup _{n} f\left(p_{n}\right)<f(p)$. We first claim that for $n$ large enough, $Y(p) \cap X\left(\operatorname{supp}\left(p_{n}\right)\right)=\emptyset$. Otherwise, we can find $x$ in $Y(p) \cap$ $\left.X\left(\operatorname{supp} p_{n}\right)\right)$ for infinitely many $n$ 's, we have $f(p)=\sum_{k} p^{k} V^{k}(x)$ and $f\left(p_{n}\right) \geq$ 
$\sum_{k} p_{n}^{k} V^{k}(x)$ for infinitely many $n$ 's, so $\lim _{\sup _{n}} f\left(p_{n}\right) \geq f(p)$ which is a contradiction. We have shown that there exists $n_{0}$ such that for $n \geq n_{0}$, $Y(p) \cap X\left(\operatorname{supp}\left(p_{n}\right)\right)=\emptyset$. If $x \in Y(p)$ and $n \geq n_{0}$, then $x \notin X\left(\operatorname{supp} p_{n}\right)$. So if $u \in \Phi(p)$ and $n \geq n_{0}$, there exists $k \in \operatorname{supp} p_{n} \backslash\{\operatorname{supp} p\}$ such that $u^{k}<0$.

Lemma 12. Let $F:[0,1] \rightrightarrows \mathbb{R}$ be a correspondence with non empty convex values and compact graph. If $F(0) \subset\{x \in \mathbb{R}, x<0\}$ and $F(1) \subset\{x \in$ $\mathbb{R}, x>0\}$, there exists $t$ in $(0,1)$ such that $0 \in F(t)$.

Proof: The sets $C_{+}=\left\{t \in[0,1], F(t) \cap \mathbb{R}_{+} \neq \emptyset\right\}$ and $C_{-}=\{t \in[0,1], F(t) \cap$ $\left.\mathbb{R}_{-} \neq \emptyset\right\}$ are closed because $F$ is u.s.c. Since $F$ has non empty values, $C_{+}$ and $C_{-}$are non empty, and $C_{+} \cup C_{-}=[0,1]$. By connexity of $[0,1]$, one can find $t$ in both sets, that is such that $F(t)$ intersects both $\mathbb{R}_{+}$and $\mathbb{R}_{-}$. Since $F(t)$ is convex, it contains 0 .

\subsubsection{Existence of an equilibrium}

For $k=2,3$, define $\delta_{k}$ as the Dirac measure on the state $k$, and $p_{-k}$ as the conditional probability on $K$ knowing the state is not $k$ :

$\delta_{2}=(0,1,0), \quad \delta_{3}=(0,0,1), \quad p_{-2}=\left(\frac{p_{1}}{p_{1}+p_{3}}, 0, \frac{p_{3}}{p_{1}+p_{3}}\right)$ and $p_{-3}=\left(\frac{p_{1}}{p_{1}+p_{2}}, \frac{p_{2}}{p_{1}+p_{2}}, 0\right)$.

Choose $u_{2}$ in $\Phi\left(\delta_{2}\right), u_{3}$ in $\Phi\left(\delta_{3}\right), u_{1,2}$ in $\Phi\left(p_{-3}\right)$ and $u_{1,3}$ in $\Phi\left(p_{-2}\right)$. These are vectors in $\mathbb{R}^{3}$, and to simply notations we write:

$$
u_{2}=\left(\begin{array}{c}
a \\
+ \\
-
\end{array}\right), u_{3}=\left(\begin{array}{c}
b \\
- \\
+
\end{array}\right), u_{1,2}=\left(\begin{array}{c}
c \geq 0 \\
+ \\
-
\end{array}\right), u_{1,3}=\left(\begin{array}{c}
d \geq 0 \\
- \\
+
\end{array}\right) .
$$

with $a=u_{2}^{1}, b=u_{3}^{1}, c=u_{1,2}^{1}$ and $d=u_{1,3}^{1}$. Here + means $\geq 0$, and - means $<0$. We have $u_{2}^{2} \geq 0, u_{3}^{3} \geq 0, c \geq 0, u_{1,2}^{2} \geq 0, d \geq 0$ and $u_{1,3}^{3} \geq 0$ since for each $p$ and $u \in \Phi(p)$, we have $u^{k} \geq 0$ for each $k \in \operatorname{supp} p$. The subset $\{2,3\}$ is not in $\mathcal{T}$, this gives $u_{2}^{3}<0, u_{3}^{2}<0, u_{1,2}^{3}<0$ and $u_{1,3}^{2}<0$.

Suppose $a \leq d$. Then a simple equilibrium exists. Player 1 uses the partition $\{\{2\},\{1,3\}\}$ to communicate: he sends the message $m=2$ if the state is 2 , and the message $m=\{1,3\}$ if the state is 1 or 3 . Player 2 proposes $x_{2}$ in $Y\left(\delta_{2}\right)$ such that $u_{2}=\left(U^{k}\left(x_{2}\right)\right)_{k \in K}$ after receiving $m=2$, and proposes $x_{1,3}$ in $Y\left(p_{-2}\right)$ such that $u_{1,3}=\left(U^{k}\left(x_{1,3}\right)\right)_{k \in K}$ after receiving $m=\{1,3\}$. By 
definition of $Y\left(\delta_{2}\right)$ and $Y\left(p_{-2}\right)$, player 2 is in best reply. And no type of player 1 has an incentive to deviate, so we have an equilibrium where player 1 plays pure. If we suppose $b \leq c$, we have a similar equilibrium where player 1 uses the partition $\{\{3\},\{1,2\}\}$.

From now on, we assume that $a>d \geq 0$ and $b>c \geq 0$. Then $a \geq 0$. Consider any sequence $\left(p_{n}\right)$ converging to the Dirac measure on state 2 such that $\operatorname{supp} p_{n}=\{1,2\}$ for each $n$. By Lemma 11 part b), we must have $\lim \sup _{n} f\left(p_{n}\right) \geq f(p)$, and since $f$ is u.s.c., $\lim \sup _{n} f\left(p_{n}\right)=f(p)$. This being true for any such sequence, $f\left(p_{n}\right) \underset{n \rightarrow \infty}{\longrightarrow} f(p)$. That is, the restriction of $f$ to the set $\{p$, supp $p \subset\{1,2\}\}$ is continuous at $\delta_{2}$. And by Lemma 11 part a), the restriction of $\Phi$ to the segment $\left[p_{-3}, \delta_{2}\right]$ has a closed graph. Similarly we have $b \geq 0$, and we can prove that the restriction of $f$ to the set $\{p$, supp $p \subset\{1,3\}\}$ is continuous at $\delta_{3}$, and the restriction of $\Phi$ to the segment $\left[p_{-2}, \delta_{3}\right]$ has a closed graph.

The initial probability $p$ is on the segment $\left[\delta_{2}, p_{-2}\right]$, and also on the segment $\left[\delta_{3}, p_{-3}\right]$. For $t \in[0,1]$, define $q_{t}=t \delta_{2}+(1-t) p_{-3}$ and $q_{t}^{\prime}$ in $\left[p_{-2}, \delta_{3}\right]$ such that $p$ belongs to the segment $\left[q_{t}, q_{t}^{\prime}\right]$. $q_{t}^{\prime}$ is uniquely defined for each $t, q_{0}^{\prime}=\delta_{3}$ and $q_{1}^{\prime}=p_{-2}$. We are going to construct an equilibrium with posteriors $q_{t}$ and $q_{t}^{\prime}$ for some appropriate $t$. We need player 1 of type 1 to be indifferent between splitting to $q_{t}$ and $q_{t}^{\prime}$.

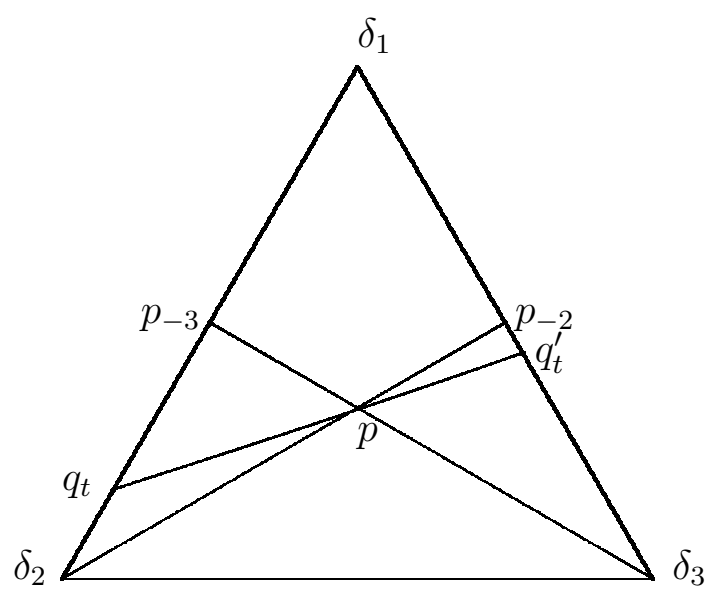


Define the correspondence $F:[0,1] \rightrightarrows \mathbb{R}$, with for each $t$ in $[0,1]$ :

$$
F(t)=\left\{u^{1}-v^{1}, u \in \Phi\left(q_{t}\right), v \in \Phi\left(q_{t}^{\prime}\right)\right\} .
$$

$F$ clearly has non empty convex compact values. We have seen that the restrictions of $\Phi$ to the segments $\left[p_{-3}, \delta_{2}\right]$ and $\left[p_{-2}, \delta_{3}\right]$ have closed graphs, moreover $q_{t}$ and $q_{t}^{\prime}$ are continuous in $t$, hence $F$ has a closed graph. $F(0)=$ $\left\{u^{1}-v^{1}, u \in \Phi\left(p_{-3}\right), v \in \Phi\left(\delta_{3}\right)\right\}$. If $F(0) \cap \mathbb{R}_{+} \neq \emptyset$, there exists a pure equilibrium where player 1 uses the partition $\{\{3\},\{1,2\}\}$, so we assume that $F(0)$ is a subset of $\{x \in \mathbb{R}, x<0\}$. Similarly, we assume that $F(1)=$ $\left\{u^{1}-v^{1}, u \in \Phi\left(\delta_{2}\right), v \in \Phi\left(p_{-2}\right)\right\}$ is a subset of $\{x \in \mathbb{R}, x>0\}$ (otherwise there exists an equilibrium where player 1 uses the partition $\{\{2\},\{1,3\}\}$ ). Then by Lemma 12 we can find $t^{*}$ in $[0,1]$ such that $0 \in F\left(t^{*}\right)$.

We can now conclude the proof. We can find $x$ in $Y\left(q_{t^{*}}\right), y$ in $Y\left(q_{t^{*}}^{\prime}\right)$, $u=\left(U^{k}(x)\right)_{k} \in \Phi\left(q_{t^{*}}\right)$ and $u^{\prime}=\left(U^{k}(y)\right)_{k} \in \Phi\left(q_{t^{*}}^{\prime}\right)$ such that for some $e \geq 0$ :

$$
u=\left(\begin{array}{c}
e \\
+ \\
-
\end{array}\right), u^{\prime}=\left(\begin{array}{c}
e \\
- \\
+
\end{array}\right)
$$

We have an equilibrium as follows. Player 1 sends a message so as to induce the posteriors $q_{t^{*}}$ and $q_{t^{*}}^{\prime}$ (type 2 sends the message 2, type 3 sends the message 3 , and type 1 randomizes between the messages 2 and 3 so that the posteriors are $q_{t^{*}}$ after $m=2$ and $q_{t^{*}}^{\prime}$ after $m=3$ ). Player 2 then proposes $x$ at $q_{t^{*}}$, and $y$ at $q_{t^{*}}^{\prime}$. Player 2 is in best reply by construction. Type 1 of player 1 is indifferent. If type 2 of player 1 deviates and sends $q_{t^{*}}^{\prime}$, player 2 will propose $y$ and type 2 will reject it, having the reserve payoff of 0 , which is not better than the payoff without deviating. Similarly, player 1 of type 3 has no profitable deviation, and we have an equilibrium. 


\section{References}

[1] Bester, H. and R. Strausz, 2001, "Contracting with imperfect commitment and the revelation principle: the single agent case," Econometrica 69, 1077-1098.

[2] Chen, Y., N. Kartik and J. Sobel, 2008, Selecting cheap talk equilibria, Econometrica 76, 117-136.

[3] Compte, O. and P. Jehiel, 2007, On quitting rights in mechanism design, American Economic Review Papers and Proceedings 97, 137-141.

[4] Compte, O. and P. Jehiel, 2009, Veto constraint in mechanism design: inefficiency with correlated types, American Economic Journal: Microeconomics 1, 182-206.

[5] Crawford, V. and J. Sobel, 1982, Strategic information transmission, Econometrica 50, 1431-1451.

[6] Forges, F., 1990, Universal mechanisms, Econometrica 58, 1341-1364.

[7] Forges, F., 1999, Ex post individually rational trading mechanisms, Current trends in Economics (edited by Alkan, A., C. Aliprantis and N. Yannelis).

[8] Forges, F. and U. Horst, 2018, Sender-receiver games with cooperation, Journal of Mathematical Economics.

[9] Gresik, T., 1991, Ex ante efficient, ex post individually rational trade, Journal of Economic Theory.

[10] Matthews, S. and A. Postlewaite, 1989, Preplay communication in twoperson sealed-bid double auctions, Journal of Economic Theory.

[11] Matthews, S., 1989, Veto threats: Rhetoric in a bargaining game, Quarterly Journal of Economics 104, 347-400.

[12] Shimizu, T. , 2013, Cheap talk with an exit option: the case of a discrete action space, Economics Letters 120, 397-400.

[13] Shimizu, T. , 2017, Cheap talk with an exit option: a model of exit and voice, International Journal of Game Theory 46, 1071-1088. 